\title{
Posttraumatic stress symptoms, depression, family functioning and children's pain in families of children with cancer: a cross-sectional study in south China
}

\author{
Yiling Yang ${ }^{1}$, Xiangyi Tan ${ }^{1}$, Ruiqing $\mathrm{Cai}^{2}$, Ping Zhang ${ }^{3}$, Yanqun $\mathrm{Hu}^{4}$, Jiangnan Meng ${ }^{3}$, \\ Jinlu Chen ${ }^{5}$, and lei shi $^{1}$ \\ ${ }^{1}$ Southern Medical University \\ ${ }^{2}$ Sun Yet-Sen University Cancer Center \\ ${ }^{3}$ Southern Medical University Nanfang Hospital \\ ${ }^{4}$ Zhujiang Hospital \\ ${ }^{5}$ Guangzhou Women and Children's Medical Center
}

September 11, 2020

\begin{abstract}
Objective: This study aimed to compare posttraumatic stress symptoms (PTSS), depression and family functioning between families of children with cancer and families of healthy children, examine the relationships among children's PTSS, parents' PTSS and depression, and family functioning, and explore the effect of disease experience on PTSS and the association between pain and PTSS in children with cancer. Methods: Participants were children with cancer $(\mathrm{n}=91)$ and their parents $(\mathrm{n}=91)$, and healthy children $(n=114)$ and their parents $(n=96)$. The children were asked to self-report PTSS and self-rate their pain degree, and the parents completed self-reported measures of PTSS, depression, and general family functioning. Results: The prevalence of severe PTSS in parents of children with cancer (21.98\%) was significantly higher than that in parents of healthy children (1.04\%). No significant difference was found in the total PTSS scores between children with cancer and healthy children. The children's PTSS was positively correlated with their parents' PTSS and depression in cancer group but not in the control group. Family functioning affected children's PTSS in both families of children with cancer and healthy families. In children with cancer, PTSS was positively correlated with pain during cancer treatment but not correlated with disease experience. Conclusions: Children with cancer perceive the fear of pain during cancer treatment, whilst their parents experience more psychological trauma. Apart from cancer treatment, attention should also be given to pain management in children with cancer and the mental health of the entire family.
\end{abstract}

\section{KEYWORDS}

Cancer, Pain, Parents, Pediatrics, Prevalence, Psychological Trauma

\section{BACKGROUND}

Pediatric cancer is a potentially life-threatening disease that severely affects the functioning of the entire family system ${ }^{1}$. Invasive procedures and painful adverse effects of the treatment increase the risk of psychological distress in children with cancer. The parents providing care for the children also feel both physically and mentally stressed, which reduces their capability to cope with new stress and may persist in their later lives $^{2}$. Children depend on their parents for both physical and emotional needs, and impaired mental health of their parents can have a negative impact on their psychological growth and physical recovery ${ }^{2}$. Currently studies remain scarce that examine the psychological distress in families of children with cancer in China. 
A previous study showed that posttraumatic stress symptoms (PTSS) were common among the parents participating in the caring of children with ongoing treatment for cancer ${ }^{4}$. A theoretical model demonstrated that PTSS of parents could affect PTSS levels in their children with cancer ${ }^{5}$. During cancer treatment, some families experience lasting damages of daily function, strained relationships and demands on family resources, which result in frequently disrupted family functioning ${ }^{6}$. Positive family functioning can protect children with cancer from developing PTSS ${ }^{7}$. About half of the individuals with posttraumatic stress disorder (PTSD) have concurrent major depressive disorder ${ }^{8}$. So far consistent findings have not been available regarding the prevalence of PTSD and depression in children with cancer and their parents. While some studies suggested

that PTSD and depression occurred in around $30 \%$ in children with cancer and their parents ${ }^{7,9,10}$, other studies reported no elevated PTSD rates or PTSS levels in either the pediatric cancer patients ${ }^{11-16}$ or their parents ${ }^{17,18}$ compared to healthy controls.

Understanding the impact of disease experience on PTSS in children with cancer may help in high risk screening for psychological stress. In children and adolescents with chronic physical illness, the association of the level of PTSS with the disease severity, duration of treatment and disease course remains elusive ${ }^{19,20}$ and needs to be defined. In addition to the psychological distress, pain is also one of the common symptoms of childhood cancer ${ }^{21,22}$, but currently how the fear of cancer pain contributes to the development of psychological trauma has not been fully explored.

The purpose of this study was to analyze the correlations among PTSS, depression and family functioning in families of children with cancer in comparison with healthy families, and explore the impact of disease experience on PTSS and the association between pain and PTSS in children with cancer. We hypothesized that (i) both parents and children in families of children with cancer had higher levels of psychological trauma than those in healthy families; (ii) the parents' PTSS and depression and family functioning were closely related to PTSS in the children; and (iii) disease experience could affect PTSS, and pain intensity was positively correlated with PTSS in children with cancer.

\section{METHODS}

\section{Study design}

This multi-centered cross-sectional study was approved by the Medical Ethics Committee of Nanfang Hospital, Southern Medical University. All the participants provided written informed consent, were fully informed of the purpose and protocol of the study, and participated in the study on a voluntary and anonymous basis. All the data collected from the participants were kept confidential to protect their privacy.

\section{Participants}

The children receiving active treatment for different types of cancer and their parents were recruited from the Department of Pediatrics of 4 general hospitals in Guangzhou, namely Pediatric Malignant Tumor Medical Centers in South China (the First Affiliated Hospital of Sun Yat-sen University), Sun Yat-sen University Cancer Center, Nanfang Hospital (Affiliated to Southern Medical University, Guangzhou, China) and Zhujiang Hospital (Affiliated to Southern Medical University). The eligible participants were between 6 and 18 years of age, had a diagnosis of cancer at least 1 month prior to the enrollment (including both newly diagnosed and recurrent cases), and had no significant cognitive or sensory deficits. Children who reported domestic violence or sexual abuse or were in a critical condition were excluded. One of the parents (father or mother) of a recruited child was invited to participate in the study, and was excluded if they had records of domestic violence or sexual abuse or had ongoing treatment for depression.

The control group consists of healthy children and their parents recruited from a public primary school in Tianhe District of Guangzhou. Specifically, one class was randomly selected from each of the fourth, fifth and sixth grades using the method of pure random numbers, and all the students in the selected class were investigated. The students who reported domestic violence or sexual abuse or had a history of serious illness were excluded. The parents of the students were selected based on the same inclusion and exclusion criteria for parents of children with cancer. 


\section{Procedure}

The investigation was conducted on a voluntary, confidential, and anonymous basis, and the participants could withdraw at any time during the study. In each of the 4 hospitals, two specially trained nurses were appointed to conduct face-to-face investigations of the participants. The questionnaires were completed by the participants in a quiet and private room. For children with difficulties in reading or comprehension, the investigators assisted them to complete the questionnaire on the basis that they fully understood the content and made their own decisions for each item. Before collection of the questionnaires, the participants were asked to re-check for omissions.

\section{Assessment of PTSS, pain, depression and family functioning}

We assessed the Children's PTSS using the Chinese version of the 22-item self-report University of California at Los Angeles PTSD Reaction Index for DSM-IV (PTSDI) ${ }^{23}$, which is designed to assess PTSS in children and adolescents aged 6-18 years. The children rated their response to the medical treatment they had received in the last month on a 5-point scale from 0 (never before) to 4 (most of the time). The total score ranges from 0 to 88 , with a higher total score indicating a greater symptom severity. A value of 38 or above on the full scale suggests a high sensitivity and specificity of PTSD diagnosis. In this study, Cronbach's $\alpha$ was 0.892 for the overall scale.

Cancer pain in the children was assessed using the Visual Analog Scale (VAS). The child marked a point on a 10-cm-long line with the two ends representing "no pain" and "unbearable pain" according to the pain severity he or she felt at that time. The length of the straight line from the starting point to the mark was measured to represent the pain severity. Study has shown that VAS is reliable and reproducible for assessing pain in children ${ }^{24}$.

We measured PTSS in the enrolled parents using the Chinese version of the 17-item self-report PostTraumatic Stress Checklist-Civilian Version (PCL-C) ${ }^{25}$. For each item, a 5-point scale from 1 (not at all) to 5 (extremely) was rated by the parents based on how much they endorsed PTSS when experiencing and witnessing their children's illness and treatment in the last month. The total score ranges from 17 to 85, with a score of 38-49 suggesting mild to moderate PTSS, a score above 50 indicating severe PTSS and possible diagnosis of PTSD. In this study, Cronbach's $\alpha$ was 0.932 for the total scale.

The depression symptoms of the parents were evaluated using the Patient Health Questionnaire Depression Scale (PHQ-9), which is a 9-item scale for simple and effective depression screening and diagnosis ${ }^{26}$. According to how they felt in the past week, the parents rated on a 4-point scale from 0 (not true at all) to 3 (almost every day). The total score ranges from 0 to 27 , and a higher score suggesting greater severity of depression. In this study, Cronbach's $\alpha$ of the total scale was 0.908 .

The family functioning was assessed using the 12-item General Functioning Sub-scale of McMaster Family Assessment Device (GF-FAD), which is a simplified version of $\mathrm{FAD}^{27}$. A 4-point scale from 1 (strongly agree) to 4 (strongly disagree) was used to rate how well each statement matched the circumstances of the family. The total score ranges from 12 to 48, with a higher score indicating a poorer family functioning. In this study, Cronbach's $\alpha$ of the total scale was 0.70 .

\section{Data analysis}

After completion of the investigation, the integrity and authenticity of the data were checked on the spot, the unqualified questionnaire (missing items, answers appeared in a certain pattern, two or more options were selected) was screened, and $50 \%$ of the collected questionnaires were randomly selected to verify the authenticity of the indicators. Data is checked and entered by two people on two machines. SPSS for Windows (Ver.20.0 IBM, New York, USA) were used to analyze the data. The demographic and clinical data of the parents and the children were presented as means with standard deviations (SDs), frequency or composition ratio. $T$ test and chi-square test were performed to examine the differences between gender and among different age groups. Pearson's Chi-square test was used to compare the prevalence of PTSS in parents and children. Independent-sample $t$ test was used to compare the total score of PCL-C, PTSDI, 
depression, and family functioning. Pearson correlation coefficient was calculated to measure the correlations among the total PCL-C score, depression, family functioning, PTSDI score and VAS score. Independentsample $t$ test and one-way analysis of variance (ANOVA) were used to examine differences in PTSDI scores among children with cancer with different disease experiences. A $P$ value less than 0.05 was considered to indicate a statistical significance.

\section{RESULTS}

\section{Participant characteristics}

A total of 120 cancer child-parent questionnaires and 120 healthy child-parent questionnaires were issued. The number of valid questionnaires recovered in cancer group is 91 (75.8\%), and $96(80 \%)$ for parents and 114 (95\%) for children in control group. Some participants did not complete the questionnaire due to changes of the children's condition, limited time, scheduling difficulties, or no interest about the research. Participants who failed to provide complete data were also excluded from the analysis. Table 1 displays the medical and demographic information for the cancer and healthy control groups. There was no significant difference in parents' $(\chi 2=1.08, \mathrm{P}=0.337 ; \mathrm{t}=-0.69, \mathrm{P}=0.49)$ and children's $\left(\chi^{2}=3.274, \mathrm{P}=0.07 ; \mathrm{t}=0.169, \mathrm{P}=0.866\right)$ gender or age between the two groups.

\section{Comparison of psychological status between families of children with cancer and healthy chil- dren}

The prevalence of PTSS in children with cancer was $8.79 \%$, significantly higher than that in healthy children $\left(0.88 \% ; \chi^{2}=7.55, P<0.01\right)$. But there was no significant difference in the total score of PTSDI between children with cancer and healthy children $(t=1.13, P=0.259)$.

The prevalence of severe PTSS was significantly higher among parents of children with cancer than those of healthy children $\left(21.98 \%\right.$ vs $\left.1.04 \% ; \chi^{2}=44.04, P<0.001\right)$. Figure 1 shows the distribution of PTSS severity in the two groups. As shown in Table 2, the scores of PCL-C, depression, and general family function were significantly higher in parents of children with cancer than those of healthy children $(t=8.96, P<0.001)$.

\section{Correlations between parents' PTSS, depression, family function and children's PTSS}

Table 3 shows that more severe PTSS and depression of the parents and a poorer general family function were associated with a higher likelihood of PTSS in the children with cancer $(r=0.317, P<0.01 ; r=0.504$, $P<0.01$; and $r=0.207, P<0.05$, respectively). Among the parents, the severity of PTSS was positively correlated with depression $(r=0.786, P<0.01)$ and family functioning $(r=0.266, P<0.05)$.

In the control group, in contrast to the cancer group, parental PTSS $(r=0.152, P>0.05)$ or depression $(r$ $=0.170, P>0.05)$ was not found to significantly correlate with PTSS in the children. But similar to the cancer group, the general family function score was positively correlated with PTSS in children $(r=0.303, P$ $<0.01)$, and parental PTSS was positively correlated with depression $(r=0.636, P<0.01)$ and general family function $(r=0.233, P<0.05)$.

Effects of disease experience on PTSS and correlations between pain and PTSS in children with cancer

We compared PTSDI scores between children with cancer who reported a history of serious illness, critical conditions, ICU admission, infections, blood transfusion, and other diseases with those who had no such history, and found no significant differences between the two groups $(P>0.05)$. We also compared PTSDI scores among children with different lengths of time after cancer diagnosis $(<6$ months, $6-12$ months, and $>12$ months), and found no significant difference among the 3 groups $(P>0.05)$. The PTSDI scores did not differ significantly between patients with disease relapse and the newly diagnosed patients $(P>0.05)$. The VAS score in children with cancer $(3.45 \pm 2.66)$ was positively correlated with their PTSDI score $(r=0.424$, $P<0.001)$.

\section{DISCUSSION}


As consistent with previous studies ${ }^{11-13}$, we found that the parents of children with cancer endorsed PTSS more than the parents of healthy children. In the parents of children with cancer, the prevalence of severe PTSS (21.98\%) in this study is comparable with that of PTSD (22\%) at 4 months after the children's diagnosis $^{28}$, but higher than that of PTSD $(17 \%)$ at 12 months or later after the diagnoses using the same scales with the same cutoff score of $50^{29}$. This discrepancy may arise from the different lengths of time after cancer diagnosis, as the parents are particularly at risk for PTSS in the first year of their children's cancer diagnosis $^{29}$. We noted that the parents of children with cancer reported more severe depression and poorer general family functioning than those of healthy children, which is consistent with previous findings ${ }^{31,32}$. A previous study found that more than $90 \%$ of families of children with cancer reported unhealthy functioning in all dimensions of FAD, with the general family function rated as the unhealthiest ${ }^{33}$. Caring for a child with cancer may trigger depression in the parents, which leads to ill family functioning.

Our results suggested that children with cancer had no elevated levels of PTSS relative to healthy children of a similar age, in contrast with the result of a previous study that reported psychological trauma resulting from childhood cancer ${ }^{7}$. But our results are supported by other studies that have included healthy controls ${ }^{11-16}$, suggesting that posttraumatic stress is less applicable in the context of psychological responses of children with ongoing cancer treatment, and being diagnosed with pediatric cancer might be considered more as a difficult but manageable stressor than a major traumatic event ${ }^{34}$. Children are too young to feel the psychological stress of cancer, while their parents may experience more psychological trauma and are at higher risks of developing severe PTSS.

In pediatric cancer families, parents' PTSS and depression were correlated with children's PTSS, which is supported by findings in pediatric oncology population ${ }^{5,35}$. However, this correlation does not exist in healthy families, possibly due to the fact that cancer families share the same stressful experience following a cancer diagnosis and during the treatment. The long and painful process of cancer treatment binds the entire family emotionally. We also found that PTSS in the parents of children with cancer was related to their depression and general family functioning, which is consistent with previous studies ${ }^{29,36}$. Poor family functioning often produces an adverse impact on the parents' psychology, and PTSS is associated with an increased risk of depression in the parents. The children's PTSS is correlated with family functioning, which underlines the importance of supporting the whole family system in the event of pediatric cancer diagnosis.

We did not find significant correlation between PTSS and disease experience in the children with cancer. Currently no relevant studies that examine the variables of disease experience have been available, and the impact of disease experience on PTSS in children with cancer still awaits further investigation by studies with larger sample sizes. In children with cancer, the severity of PTSS is reported to be independent of the treatment responses or the length of time after diagnosis ${ }^{19}$. PTSS is associated more with such personality factors and traits as adaptive style, rather than the history of health conditions ${ }^{14}$. Presumably, the disease experience increases the vulnerability of the children to life challenges instead of directly leading to PTSS.

Pain reported by children with cancer is related to $\mathrm{PTSS}^{37}$, but the nature of this relationship remains unclear. Children normally do not fully understand the significance of a cancer diagnosis, but the pain caused by cancer treatment has an intuitive psychological impact. Children are more sensitive to the feeling of pain than to the disease experience of cancer, suggesting the importance of effective pain management in order to alleviate the traumatic experiences. Patient-controlled analgesia or authorized agent-controlled analgesia is safe, timely, and acceptable to children, which can achieve the best balance between effective pain management and adverse reactions ${ }^{38}$.

\section{Study limitations}

Some limitations to this study should be noted. First, this cross-sectional study does not support a conclusion of causality, which shall be explored by future studies of a longitudinal design at the family system level; nor is it impossible to determine the directionality of the relationship among children's PTSS, parents' PTSS and depression, and family functioning. Second, only self-reported screening tools were used in this study to measure PTSS. Nevertheless, the purpose of this study is to screen PTSS and determine the severity of PTSS, 
and the measurement tools have good reliability and validity. Finally, less than $80 \%$ of valid questionnaires were recovered in cancer group, indicating that families of children with cancer did not have time to care for anything other than the treatment of the child, and that this group urgently needed adequate psychosocial support.

\section{Clinical implications}

Pain caused by treatment is closely related to psychological trauma of children with cancer. Effective pain management and relieving the physical and psychological symptoms will help to improve children's quality of life. The mental health of the parents directly affects the children's mental health and the general family functioning. Early detection of the psychological trauma of the parents and timely interventions can have a far-reaching positive impact on the prognosis of the children. Application of the techniques from the cognitive-behavioral theoretical framework is recommended for the family members of children with cancer ${ }^{39}$. For parents of children with ongoing cancer treatment, intervention with Internet-based guided self-help following the principles of cognitive behavior therapy has shown promising effects for relieving PTSS and related symptoms ${ }^{40}$.

Conclusions

The parents of children with cancer have more severe PTSS and depression than the parents of healthy children, but the children with cancer and healthy children have comparable PTSS level. Disease experience of the children with cancer does not obviously affect the development of PTSS, while pain experienced during cancer treatment may trigger PTSS. The parents' PTSS and depression, family functioning and children's PTSS are closely related in families of children with cancer. Supportive psychological intervention is strongly recommended for families of children with cancer.

\section{ACKNOWLEDGEMENTS}

The work was supported by the Natural Science Foundation of Guangdong Province, China (Grant Number 2018030310417).

\section{CONFLICT OF INTEREST STATEMENT}

The authors declare no conflict of interest.

\section{DATA AVAILABILITY STATEMENT}

The data that support the findings of this study are available on request from the corresponding author. The data are not publicly available due to privacy or ethical restrictions.

\section{References:}

1. Ward E, DeSantis C, Robbins A, Kohler B, Jemal A. Childhood and adolescent cancer statistics, 2014. CA Cancer J Clin . 2014;64(2):83-103

2. Yeh CH. Dynamic coping behaviors and process of parental response to child's cancer. Appl Nurs Res . $2003 ; 16(4): 245-55$

3. Darling SJ, Hearps S, Muscara F et al. Psychological trajectories of mothers and fathers following their child's diagnosis of a life-threatening illness or injury: A longitudinal investigation. J Clin Psychol . 2019;75(10):1930-1942

4. Kazak AE, Boeving CA, Alderfer MA, Hwang WT, Reilly A. Posttraumatic stress symptoms during treatment in parents of children with cancer. J Clin Oncol . 2005;23(30):7405-10

5. Goldwin M, Lee S, Afzal K, Drossos T, Karnik N. The Relationship between Patient and Parent Posttraumatic Stress in Pediatric Oncology: A Theoretical Framework. Child Health Care . 2014;43(1):1-15

6. Long KA, Marsland AL. Family adjustment to childhood cancer: a systematic review. Clin Child Fam Psychol Rev . 2011;14(1):57-88 
7. Bruce M. A systematic and conceptual review of posttraumatic stress in childhood cancer survivors and their parents. Clin Psychol Rev . 2006;26(3):233-56

8. Flory JD, Yehuda R. Comorbidity between post-traumatic stress disorder and major depressive disorder: alternative explanations and treatment considerations. Dialogues Clin Neurosci . 2015;17(2):141-50

9. van Warmerdam J, Zabih V, Kurdyak P et al. Prevalence of anxiety, depression, and posttraumatic stress disorder in parents of children with cancer: A meta-analysis. Pediatr Blood Cancer . 2019;66(6):e27677

10. Shi L, Gao Y, Zhao J et al. Prevalence and predictors of posttraumatic stress symptoms in parents of children with ongoing treatment for cancer in South China: a multi-centered cross-sectional study. Support Care Cancer . 2017;25(4):1159-1167

11. Barakat LP, Kazak AE, Meadows AT et al. Families surviving childhood cancer: a comparison of posttraumatic stress symptoms with families of healthy children. J Pediatr Psychol . 1997;22(6):843-59

12. Kazak AE, Barakat LP, Meeske K et al. Posttraumatic stress, family functioning, and social support in survivors of childhood leukemia and their mothers and fathers. J Consult Clin Psychol . 1997;65(1):120-9

13. Brown RT, Madan-Swain A, Lambert R. Posttraumatic stress symptoms in adolescent survivors of childhood cancer and their mothers. J Trauma Stress . 2003;16(4):309-18

14. Phipps S, Jurbergs N, Long A. Symptoms of post-traumatic stress in children with cancer: does personality trump health status? Psychooncology . 2009;18(9):992-1002

15. Phipps S, Klosky JL, Long A et al. Posttraumatic stress and psychological growth in children with cancer: has the traumatic impact of cancer been overestimated? J Clin Oncol . 2014;32(7):641-6

16. Tillery R, Willard VW, Long A, Phipps S. Posttraumatic stress in young children with cancer: Risk factors and comparison with healthy peers.Pediatr Blood Cancer . 2019;66(8):e27775

17. Jurbergs N, Long A, Ticona L, Phipps S. Symptoms of posttraumatic stress in parents of children with cancer: are they elevated relative to parents of healthy children? J Pediatr Psychol . 2009;34(1):4-13

18. Phipps S, Long A, Willard VW et al. Parents of Children With Cancer: At-Risk or Resilient? J Pediatr Psychol . 2015;40(9):914-25

19. Currier JM, Jobe-Shields LE, Phipps S. Stressful life events and posttraumatic stress symptoms in children with cancer. J Trauma Stress . 2009;22(1):28-35

20. Pinquart M. Posttraumatic Stress Symptoms and Disorders in Parents of Children and Adolescents With Chronic Physical Illnesses: A Meta-Analysis.J Trauma Stress . 2019;32(1):88-96

21. Allen JM, Graef DM, Ehrentraut JH, Tynes BL, Crabtree VM. Sleep and Pain in Pediatric Illness: A Conceptual Review. Cns Neurosci Ther . 2016;22(11):880-893

22. Tutelman PR, Chambers CT, Stinson JN et al. Pain in Children With Cancer: Prevalence, Characteristics, and Parent Management. Clin J Pain . 2018;34(3):198-206

23. Steinberg AM, Brymer MJ, Decker KB, Pynoos RS. The University of California at Los Angeles Posttraumatic Stress Disorder Reaction Index. Curr Psychiatry Rep . 2004;6(2):96-100

24. Bailey B, Gravel J, Daoust R. Reliability of the visual analog scale in children with acute pain in the emergency department. Pain . 2012;153(4):839-42

25. Ruggiero KJ, Del BK, Scotti JR, Rabalais AE. Psychometric properties of the PTSD Checklist-Civilian Version. J Trauma Stress . 2003;16(5):495-502

26. Spitzer RL, Kroenke K, Williams JB. Validation and utility of a self-report version of PRIME-MD: the PHQ primary care study. Primary Care Evaluation of Mental Disorders. Patient Health Questionnaire. JAMA . 1999;282(18):1737-44 
27. Byles J, Byrne C, Boyle MH, Offord DR. Ontario Child Health Study: reliability and validity of the general functioning subscale of the McMaster Family Assessment Device. Fam Process . 1988;27(1):97-104

28. Pöder U, Ljungman G, von Essen L. Posttraumatic stress disorder among parents of children on cancer treatment: a longitudinal study. Psychooncology . 2008;17(5):430-7

29. Stoppelbein L, Greening L. Brief report: the risk of posttraumatic stress disorder in mothers of children diagnosed with pediatric cancer and type I diabetes. J Pediatr Psychol . 2007;32(2):223-9

30. Pai AL, Greenley RN, Lewandowski A et al. A meta-analytic review of the influence of pediatric cancer on parent and family functioning. J Fam Psychol . 2007;21(3):407-15

31. Sharghi A, Karbakhsh M, Nabaei B, Meysamie A, Farrokhi A. Depression in mothers of children with thalassemia or blood malignancies: a study from Iran. Clin Pract Epidemiol Ment Health . 2006;2:27

32. Fotiadou M, Barlow JH, Powell LA, Langton H. Optimism and psychological well-being among parents of children with cancer: an exploratory study.Psychooncology . 2008;17(4):401-9

33. Modanloo S, Rohani C, Shirinabadi FA, Vasli P, Pourhosseingholi A. General Family Functioning as a Predictor of Quality of Life in Parents of Children With Cancer. J Pediatr Nurs . 2019;44:e2-e8

34. Sharp K, Lindwall JJ, Willard VW et al. Cancer as a stressful life event: Perceptions of children with cancer and their peers. Cancer-Am Cancer Soc . 2017;123(17):3385-3393

35. Bakula DM, Sharkey CM, Perez MN et al. Featured Article: The Relationship Between Parent and Child Distress in Pediatric Cancer: A Meta-Analysis.J Pediatr Psychol . 2019;44(10):1121-1136

36. Muscara F, McCarthy MC, Thompson EJ et al. Psychosocial, Demographic, and Illness-Related Factors Associated With Acute Traumatic Stress Responses in Parents of Children With a Serious Illness or Injury. J Trauma Stress . 2017;30(3):237-244

37. Pöder U, Ljungman G, von Essen L. Parents' perceptions of their children's cancer-related symptoms during treatment: a prospective, longitudinal study. J Pain Symptom Manage . 2010;40(5):661-70

38. Cooney MF, Czarnecki M, Dunwoody C et al. American Society for Pain Management Nursing position statement with clinical practice guidelines: authorized agent controlled analgesia. Pain Manag Nurs . 2013;14(3):176-81

39. Sánchez-Egea R, Rubio-Aparicio M, Sánchez-Meca J, Rosa-Alcázar AI. Psychological treatment for family members of children with cancer: A systematic review and meta-analysis. Psychooncology . 2019;28(5):960969

40. Cernvall M, Carlbring P, Ljungman L, Ljungman G, von Essen L. Internet-based guided self-help for parents of children on cancer treatment: a randomized controlled trial. Psychooncology . 2015;24(9):1152-8

\section{List of table/figure legends:}

Tables:

Table 1. Demographic and clinical characteristics of the parents and their children participating in this study

Table 2. Descriptive statistics for PTSS, depression, and general family function

Table 3. Correlations for Parental PTSS, Depression, General family function, and Child's PTSS

Figures:

Figure 1. Differences in proportion of self-reported PTSS severity in parents of children with cancer and parents of healthy children

Hosted file 
TABLE 1.docx available at https://authorea.com/users/357160/articles/479848-posttraumaticstress-symptoms-depression-family-functioning-and-children-s-pain-in-families-ofchildren-with-cancer-a-cross-sectional-study-in-south-china

\section{Hosted file}

TABLE 2.docx available at https://authorea.com/users/357160/articles/479848-posttraumaticstress-symptoms-depression-family-functioning-and-children-s-pain-in-families-ofchildren-with-cancer-a-cross-sectional-study-in-south-china

\section{Hosted file}

TABLE 3.docx available at https://authorea.com/users/357160/articles/479848-posttraumaticstress-symptoms-depression-family-functioning-and-children-s-pain-in-families-ofchildren-with-cancer-a-cross-sectional-study-in-south-china

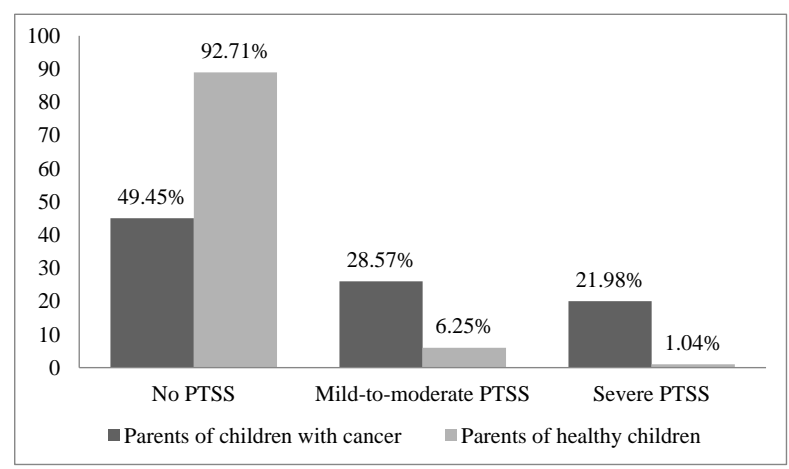

FIGURE 1 Differences in proportion of self-reported PTSS severity in parents of

children with cancer and parents of healthy children 